\title{
Efficacy and mechanism of KRAS G12C PROTAC in inhibiting the proliferation of pancreatic cancer cells
}

\author{
Shuai Gao ${ }^{1}$ Fangxia Zou $^{1}$ Lixia Zheng $^{2}$ Yunjie Wang $^{1}$ Xinyu Feng $^{1}$ Deshuai Liu $^{1}$ Yutong Mao $^{1}$ Liang Ye $^{3^{*}}$ Jingwei Tian $^{1}$ \\ ${ }^{1}$ College of Pharmacy, Yantai University, Yantai 264000, Shandong Province, China \\ ${ }^{2}$ Shandong Luye Pharmaceutical Co. Ltd., Yantai 264000, Shandong Province, China \\ ${ }^{3}$ School of Public Health and Management, Binzhou Medical University, Yantai 264000, Shandong Province, China
}

\section{Check for updates}

Correspondence to: (1) Liang Ye, School of Public Health and Management, Binzhou Medical University, Yantai 264000, Shandong Province, China;

Email: yeliang@luye.cn;

(2) Jingwei Tian, College of Pharmacy, Yantai University, Yantai 264000, Shandong Province, China;

Email: tianjingwei@luye.com

Received: December 24, 2021;

Accepted: January 8, 2022;

Published: January 11, 2022.

Citation: Gao S, Zou F, Zheng L, et al. Efficacy and mechanism of KRAS G12C PROTAC in inhibiting the proliferation of pancreatic cancer cells. J Pharm Biopharm Res, 2022, 3(1): 176-184.

https://doi.org/10.25082/JPBR.2021.01.002

Copyright: () 2022 Shuai Gao et al. This is an open access article distributed under the terms of the Creative Commons Attribution License, which permits unrestricted use, distribution, and reproduction in any medium, provided the original author and source are credited.

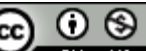

\begin{abstract}
Pancreatic cancer is a rare but highly malignant cancer with few effective treatments available. Targeting cancers bearing specific genetic mutations offers a new approach for cancer therapy. PROTAC (proteolysis-targeting chimeras) is an emerging technique to design targeted therapy and increasing evidence supports its utility. This study examined the in vitro pharmacodynamics and mechanism of PROTAC K-Ras Degrader-1 (PKD-1), a PROTAC molecule, in inhibiting the proliferation of pancreatic cancer cells. We used a pancreatic cancer cell line, MIA PaCa-2 cells, to examined the binding and degradation-promoting capabilities of PKD-1 on KRAS G12C protein and further evaluated the effects of PKD-1 on cell viability, cell cycle and apoptosis. PKD-1 was able to bind to KRAS G12C protein, promoted its degradation for up to $72 \mathrm{~h}$, reduced cell viability, increased cell cycle arrest and promoted cell apoptosis. Mechanistic study found that the efficacy of PKD-1 was at least partially mediated by promoting $26 \mathrm{~S}$ proteasome degradation process. Combined, these results extended previous findings and support the potential utility of PROTAC molecules such as PKD-1 as a new treatment strategy against pancreatic cancer.
\end{abstract}

Keywords: KRAS, KRAS G12C, PROTAC K-Ras Degrader-1, pancreatic cancer, apoptosis

\section{Introduction}

Pancreatic cancer is a relatively uncommon malignancy with a very high mortality rate, occurring mainly in people aged 40 years and older $[1,2]$ and the health burden of pancreatic cancer is increasing in most countries. An estimated 447,665 new cases of pancreatic cancer were recorded globally in 2017 (58.6 per million) and this number was likely an underestimate. In the US, approximately 60430 was newly diagnosed in 2021. Incidence, prevalence and mortality increased by 55\%, 63\% and 53\% in the past 25 years, respectively. Pancreatic cancer accounts for $1.8 \%$ of all cancers, causing $3.9 \%$ of all cancer disability-adjusted life years and $4.6 \%$ of all cancer deaths [3]. Effective screening is not available for pancreatic cancer and most patients present with locally advanced (30-35\%) or metastatic (50-55\%) disease at diagnosis [4]. Despite great efforts have been undertaken to treat diagnosed pancreatic cancer with surgery, radiation and adjuvant chemotherapies, the overall survival rate remains abysmal with 5 year overall survival rate lingering around 10\% [4,5]. Novel and more targeted therapies are in dire need to radically improve the treatment results of pancreatic cancer. Recent large-scale molecular profiling efforts have attempted to uncover genomic subtype prevalence and corresponding therapeutic relevance in pancreatic cancer. One such effort found that the mutations in the RAS gene are present in about $90 \%$ of patients with pancreatic cancer [6], and as a result therapies that target the RAS gene may be an effective way to improve pancreatic cancer treatment results.

The rat sarcoma (RAS) gene was the first identified proto-oncogene and functionally acquired RAS mutations occur in approximately $30 \%$ of human cancers. The most common RAS mutations include Kirsten RAS (KRAS), Harvey RAS (HRAS) and neuroblastoma RAS (NRAS), with KRAS being the predominant mutant subtype, accounting for approximately $85 \%$ of all RAS mutations. The KRAS mutations are genetic drivers of multiple cancer types, especially colorectal cancer, pancreatic cancer and non-small cell lung cancer [7]. KRAS mutations at codon 12 (KRAS-G12) account for $89 \%$ of KRAS mutations in human cancers, among which three most common mutations are G12C, G12D, and G12V. KRAS G12C, a small GTPase, switches between a GTP-bound active state and a GDP-bound inactive state, and the transition 
between these states is regulated by guanine nucleotide-exchange factors (GEFs) which promote the exchange of GDP to GTP and GTPase-activating protein (GAP) which favor the exchange of GTP to GDP [8]. RAS proteins had long been considered "non-druggable targets" due to the lack of ideal binding pockets on their surface; however, recent studies have revealed that the KRAS G12C protein has two superficial binding pockets, Switch I and Switch II, on its surface. Small molecule compounds can form irreversible covalent binding to cysteines in the Switch II binding pocket, locking KRAS into an inactive state and thereby inhibiting activation of downstream pathways. For example, PROTAC (PROteolysis TArgeting Chimeras) molecules can bind to KRAS G12C proteins and affect the circulation of GTP and GDP, thus inhibiting the signaling pathway and slowing down the proliferation and migration of tumor cells [5]. In May 2021, Sotorasib (AMG-510), a covalently bound small molecule targeting KRAS G12C has been approved by the FDA for the treatment of locally advanced or metastatic non-small cell lung cancer in adult patients with KRAS G12C mutation [9]. Another compound with similar mechanism of action with Sotorasib, Adagrasib (MRTX-849), is currently in a clinical phase III study [10]. However, both Adagrasib and Sotorasib have been reported to develop acquired drug resistance and MAPK pathway reactivation during dosing [11]. Therefore, it seems important and necessary to find new therapeutic strategies for targeted therapy of KRAS mutated cancers. PROTAC technology is a protein degradation technique in which the PROTAC molecule contains three important components, namely an E3 ubiquitin ligase ligand, a target protein of interest (POI) ligand and a linker connecting the two ligands [12]. PROTAC molecule links the target protein to the $\mathrm{E} 3$ ubiquitinase and subsequently ubiquitinates the target protein, thereby allowing it to enter the protease pathway for degradation [13]. The PROTAC small molecule ARV-110, which targets androgen receptors, has entered clinical phase II trial for the treatment of prostate cancer [14], suggesting that PROTAC technology can be a viable approach to target and degrade KRAS G12C for the treatment of pancreatic cancer.

PROTAC K-Ras Degrader-1 (PKD-1) is a potent KRAS G12C degrader by linking the E3 ligase ligand CRBN to a small molecule KRAS G12-targeting compound via a segment of linker which demonstrates $>70 \%$ degradation efficacy in SW1573 cells, a human alveolar cell carcinoma cell line bearing KRAS G12C mutation [15]. In this study, we evaluated the ability of PROTAC K-Ras Degrader-1 to bind to KRAS G12C protein and determined the effects of PKD-1 on cell proliferation activity, cell cycle and apoptosis. Our results demonstrated that PROTAC K-Ras Degrader-1 had the ability to target and degrade KRAS G12C protein, inhibit cell proliferation by blocking the cell cycle in G0/G1 phase, and subsequently promote apoptosis. These results provided the first line of evidence supporting the usefulness of PROTAC K-Ras Degrader-1 against pancreatic cancer.

\section{Materials and methods}

\subsection{Primary instruments}

Ultra Clean Bench (Shanghai Shangjing, China); $\mathrm{CO}_{2}$ Incubator (SANYO, Japan); OLYMPUSIX73 Inverted Fluorescence Microscope (Olympus, Beijing, China); Centrifuge (Beckman, USA); Enzyme Labeler (Burton Instruments, USA); Automatic Chemiluminescence Imaging Gel Imaging System (Beijing Saizhi Venture Technology Co., Ltd.); Flow Cytometer (Bio-Rad USA).

\subsection{Drugs and reagents}

$\beta$-actin antibody (AA128-1), RPS6 (AF7917), phosphorylated RPS6 (AF5917), ERK1/2 (AF1051), phosphorylated ERK1/2 (AF5902), enzyme-labeled goat anti-rabbit IgG (A0208), enzyme-labeled goat anti-mouse IgG (A0216), Hoechst staining kit (C0003), and BCA concentration determination kit (P0010) were purchased from Beyotime Biotechnology Co. (Shanghai, China). KRAS G12C Nucleotide Exchange Assay Kit (\#79859) was purchased from BPS Bioscience (San Diego, CA, USA). ATP Lite 1-STEP Luminescence Assay System (6016731) was purchased from PerkinElmer (Massachusetts, USA). Cell Cycle Assay Kit (Kga512) was purchased from KeyGen Biotech (Nanjing, China). PROTAC K-Ras Degrader-1 (PKD-1) (\#Hy-129523) and MRTX-849 (\#Hy-130149) were purchased from MedChemExpress (Monmouth Junction, NJ, USA). Bortezomib Bortezomib (PS-341) (\#L2007282) was purchased from Aladdin Bio-Chem Technology (Shanghai, China). PKD-1 and MRTX-849 were dissolved in DMSO and stored at $-20^{\circ} \mathrm{C}$ for less than 1 month before use in in vitro experiments. Vehicle (DMSO) was used as a control in all experiments at a maximum concentration of $0.1 \%$.

The chemical structure of PROTAC K-Ras Degrader-1 (PKD-1) is shown in Figure 1. 


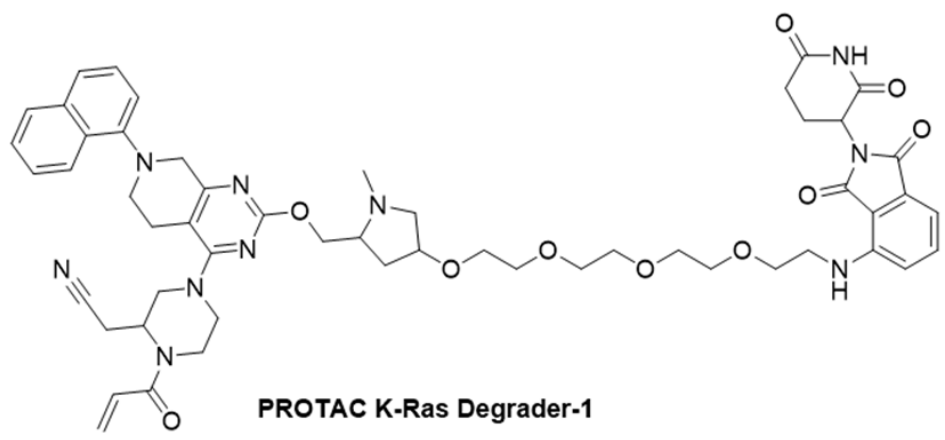

Figure 1 Chemical structure of PKD-1

\subsection{Cell lines and culture}

The human pancreatic cancer cell line MIA PaCa-2 was purchased from the Stem Cell Bank of the Chinese Academy of Sciences (Shanghai, China) and grown in Dulbecco's modified Eagle's medium (Gibco, Grand Island, NY, USA) supplemented with $10 \%$ fetal bovine serum (FBS) (Sijiqing Biological Engineering Materials, Hangzhou, China), $100 \mathrm{U} / \mathrm{ml}$ penicillin and $100 \mathrm{mg} / \mathrm{ml}$ streptomycin at $37^{\circ} \mathrm{C}$ with $5 \% \mathrm{CO}_{2}$. All cells were harvested in the exponential growth phase for assays.

\subsection{KRAS G12C nucleotide exchange assay}

To examine the binding ability of PKD-1 to KRAS G12C protein, the KRAS G12C nucleotide exchange assay kit was used and the assay was performed according to the protocol guidance. Briefly, prepare $2 \times$ KRAS (G12C) buffer, DTT, and BODIPY-GDP loaded with KRAS G12C, along with $1 \times$ KRAS G12C buffer containing $1 \mathrm{mmol} / \mathrm{L}$ DTT and $15 \mu \mathrm{L}$ of the required primary reaction solution (obtained by mixing $5 \mu \mathrm{L}$ of BODIPY-GDP with $10 \mu \mathrm{L}$ of $1 \times$ KRAS G12C buffer), and add to each well $15 \mu \mathrm{L}$ of the primary reaction solution. A solution of PKD-1 and MRTX-849 containing 5\% DMSO was prepared, and a concentration gradient between $0.0005-10 \mu \mathrm{mol} / \mathrm{L}$ was used. $5 \mu \mathrm{L}$ of sample was added to each well and centrifuge to ensure that the samples were well mixed, and the samples were then incubated at room temperature for $2 \mathrm{~h}$. The samples were then mixed at a 1:1 ratio of GTP $(10 \mu \mathrm{M})$ and EDTA $(25 \mathrm{mM})$ solutions and incubated at room temperature for $2 \mathrm{~h}$. After incubation, $5 \mu \mathrm{L}$ of the GTP/EDTA mixture was added to initiate the reaction and incubated at room temperature for $1 \mathrm{~h}$. Then the fluorescence at Ex470 nm/Em $525 \mathrm{~nm}$ was measured.

\subsection{Adenosine triphosphate (ATP) assay}

ATP assay (ATPlite 1 step Luminescence Assay System, PerkinElmer, Waltham, MA, USA) was used to measure cell proliferation viability and the effect of PKD-1 on cell proliferation activity according to manufacturer's instructions. Briefly, MIA PaCa- 2 cells were suspended to $1 \times 10^{3}$ cells $/ \mathrm{mL}$, inoculated with $90 \mu \mathrm{L}$ per well in a black opaque 96 -well plate and incubated overnight at $37^{\circ} \mathrm{C}$ in a $5 \% \mathrm{CO}_{2}$ cell incubator. The next day the cells were treated with different concentrations of PKD-1 or MRTX-849 and the control group was treated with DMEM culture medium. PKD-1 and MRTX-849 stock solutions were prepared at a concentration of $10 \mathrm{mmol} / \mathrm{L}$, respectively, and diluted with DMEM culture medium to a concentration gradient $(0.0005-10$ $\mu \mathrm{mol} / \mathrm{L}$ ) and added to the culture wells at $10 \mu \mathrm{L}$ each. An equal volume of DMEM culture solution was added to the control group. The 96-well plates were incubated for $72 \mathrm{~h}$ at $37^{\circ} \mathrm{C}$ in a $5 \% \mathrm{CO}_{2}$ incubator. $100 \mu \mathrm{L}$ of ATP LITE 1-STEP solution was added to each well in a light-protected environment, and the luminescence intensity of each well was determined by shaking for $5 \mathrm{~min}$ with a microshaker. The cells treated with DMEM medium were used as the control group, and the half-inhibitory concentration $\left(\mathrm{IC}_{50}\right)$ was calculated.

The effect of PS-341 on cell proliferation activity was also assessed by the ATP method, and a concentration of PS-341 that was not toxic to cells was selected for the experiment. 96-well plates were prepared and DMEM medium containing PS-341 at final concentrations of 10 and 40 $\mathrm{nmol} / \mathrm{L}$ was added, respectively, and the control group was treated with DMEM medium at 100 $\mu \mathrm{L}$ per well for $24 \mathrm{~h}$. After $24 \mathrm{~h}$, the culture medium was replaced, and the final concentrations of PS-341 remained at 10 and $40 \mathrm{nmol} / \mathrm{L}$, respectively, while the final concentration gradients of PKD-1 were $0.0015-10 \mu \mathrm{mol} / \mathrm{L}$. The cells were further incubated for $72 \mathrm{~h}$ at $37^{\circ} \mathrm{C}$ in a $5 \%$ $\mathrm{CO}_{2}$ incubator, and then ATP assay was performed as described above. 


\subsection{Western blotting assay}

Effects of PKD-1 on KRAS degradation was examined using Western blotting assay. For this assay, total protein was extracted from cells and examined following previous published protocols [16]. Briefly, cells were lysed in the RIPA buffer and centrifuged at $4{ }^{\circ} \mathrm{C}$. Equal amounts of protein (20-35 $\mu \mathrm{g})$ were separated with SDS-PAGE and transferred onto PVDF membranes. Membranes were blocked and then incubated overnight with primary antibodies. After washes, the membranes were incubated with secondary antibodies. The immunoreactive bands were visualized using BeyoECL Plus purchased from Beyotime Biotechnology Co. (Shanghai, China) by enhanced chemiluminescence system (Pittsburgh, PA, USA).

\subsection{Flow cytometry}

Cell cycle analysis was performed using flow cytometry (Bio-Rad Laboratories, Hercules, CA USA) following previously published protocol [17]. Briefly, the MIA PaCa-2 cells were seeded into 6-well plates with a density of $1 \times 10^{5}$ cells/well to adhere overnight, followed by treatment with 0.1 and $1 \mu \mathrm{mol} / \mathrm{L}$ of PKD- 1 or DMEM medium for $24 \mathrm{~h}$. The effects of PKD-1 on cell cycle progression and apoptosis were determined using fluorescence-activated cell sorting (FACS) analysis with PI or PI/FITC-labeled annexin stained cells according to the manufacturer's recommended procedures and analyzed by the flow cytometer.

\subsection{Apoptosis assay}

MIA PaCa- 2 cells were cultured in 6-well plates at $3 \times 10^{5}$ cells/well and incubated with PKD- 1 at 0.1 and $1 \mu \mathrm{mol} / \mathrm{L}$ for $24 \mathrm{~h}$ to explore a dose-dependent analysis. Harvested cells were washed with PBS and stained with Annexin V and Propidium Iodide (Beyotime, Shanghai, China). Cells were examined by a fluorescence microscopy (Olympus, Tokyo, Japan).

\subsection{Statistical analysis}

Data were expressed as mean $\pm \mathrm{SD}$ and analyzed using one-way analysis of variance followed by Dunnett's test. Statistical analysis was performed using SPSS 26.0 software, and $\mathrm{P}<0.05$ was considered statistically significant.

\section{Results}

\subsection{PKD1 binding to KRAS G12C protein}

To examine the binding ability of PKD-1 to KRAS G12C protein, the KRAS G12C nucleotide exchange assay was used. As shown in Figure 2, PKD-1 immobilized KRAS G12C in the inactive state, and the fluorescence intensity increased with increasing drug concentration, indicating that PKD-1 could effectively bind to KRAS G12C protein. The $\mathrm{EC}_{50}$ values of PKD1 and MRTX-849 bound to KRAS G12C protein were 0.1447 and $0.1973 \mu \mathrm{mol} / \mathrm{L}$, respectively, suggesting that PKD-1 and MRTX-849 have similar binding affinity to KRAS G12C protein.

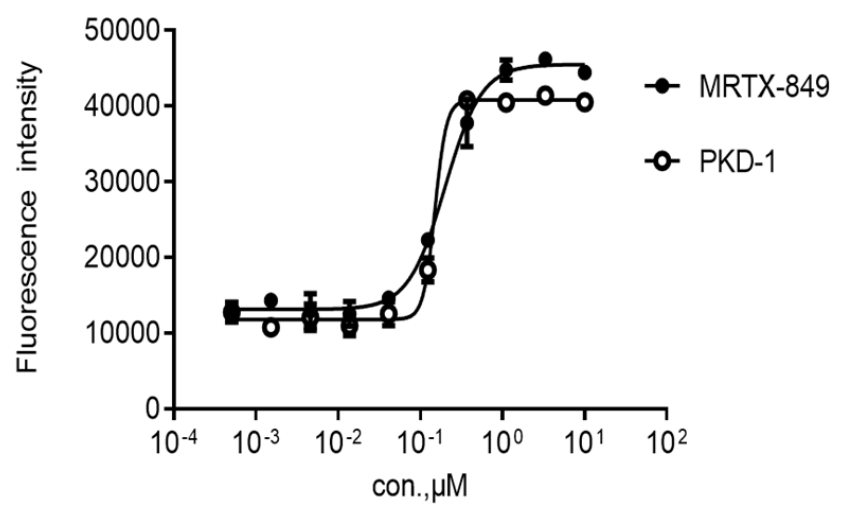

Figure 2 Protein binding experiment

The binding ability of PKD-1 to KRAS G12C protein was further examined by Western blotting assay. As shown in Figure 3, with the increase of PKD-1 concentration, the KRAS molecular weight was increased and the KRAS protein bands shifted upward, suggesting that 
PKD-1 bond directly to KRAS G12C protein and as a result the compound increased the molecular weight of KRAS.

A.

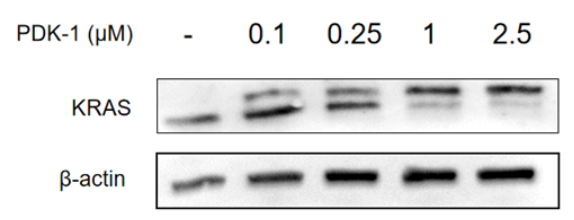

B.

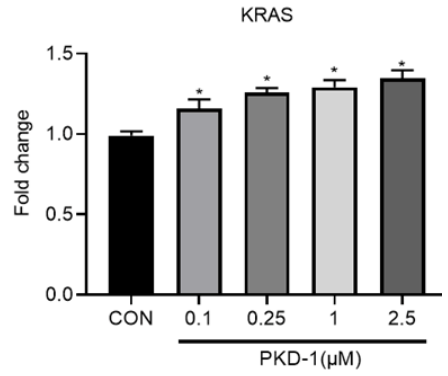

Figure 3 Binding of PKD-1 with KRAS protein; A, B Western blotting analysis of the expression of KRAS in MIA PaCa- 2 cells treated with PKD-1 $(0.1,0.25,1$, and $2.5 \mu \mathrm{mol} / \mathrm{L})$ for $8 \mathrm{~h}$; The data are expressed as the mean $\pm \mathrm{SD}(\mathrm{n}=3)$; Compared with the control group, ${ }^{*} p<$ 0.05 .

\subsection{PKD-1 promotes the degradation of KRAS G12C protein}

Because PKD-1 was able to directly bind to KRAS G12C (Figure 3), next we examined whether the binding of PKD-1 could promote the degradation of KRAS G12C protein. A binding time course study showed that at a fixed concentration of $0.5 \mu \mathrm{mol} / \mathrm{L}, \mathrm{PKD}-1$ first time-dependently shifted the KRAS G12C protein band upward, and then promoted KRAS G12C degradation starting from $24 \mathrm{~h}$ onward (Figure 4). This result indicates that PKD-1 binding on KRAS protein promoted the degradation of KRAS protein.

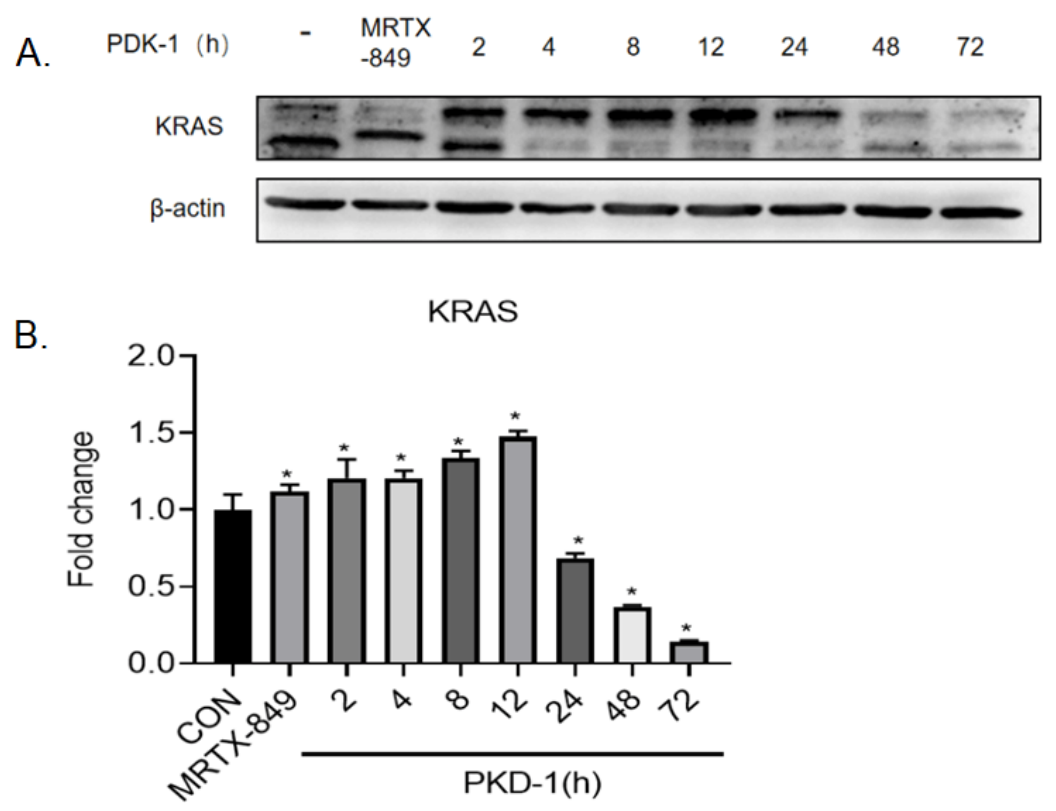

Figure 4 PKD-1 promoted the degradation of KRAS G12C protein; A, B Western blotting analysis of the expression of KRAS in MIA PaCa- 2 cells treated with PKD-1 or MRTX-849 $(2,4,8,12,24,48,72 \mathrm{~h})$ for $0.5 \mu \mathrm{mol} / \mathrm{L}$; The data are expressed as the mean $\pm \mathrm{SD}(\mathrm{n}=3)$. Compared with the control group, ${ }^{*} p<0.05$.

\subsection{Effect of PKD-1 on cell proliferation activity}

Given that PKD-1 was able to promote the degradation of KRAS G12C protein (Figure 4), we next examined whether this effect could lead to the inhibition of MIA PaCa-2 cell proliferation. As shown in Figure 5, both PKD-1 and MRTX-849 significantly and concentration-dependently inhibited the proliferation of MIA PaCa- 2 cells. The IC $_{50}$ values of PKD-1 and MRTX-849 on MIA PaCa- 2 cell viability were 0.276 and $0.005 \mu \mathrm{mol} / \mathrm{L}$, respectively, suggesting that MRTX-849 was more potent than PKD-1 in this assay. For MRTX-849, the inhibitory effect was already quite pronounced at the lowest concentration tested. At higher concentrations, the 
inhibitory effect of PKD-1 and MRTX-849 was similar and both were very effective against cell proliferation.

MIA PaCa-2

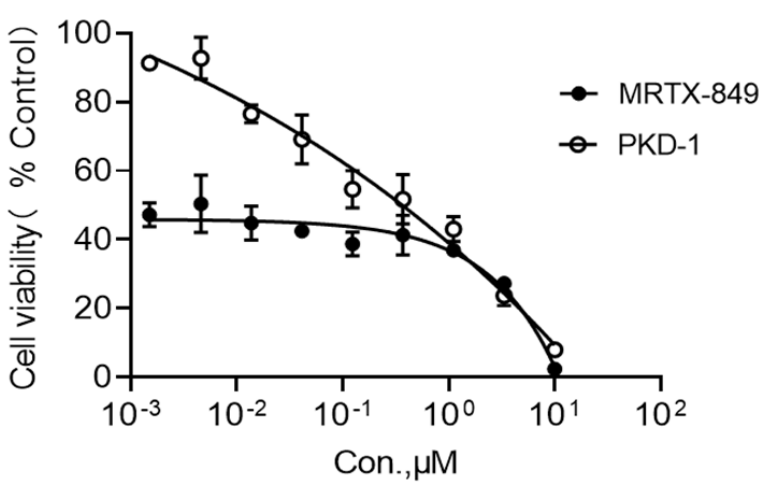

Figure 5 Effects of PKD-1 and MRTX-849 on cell proliferation

\subsection{Role of proteasome inhibition in PKD-1-induced inhibition of cell proliferation}

Because the mechanism of PROTAC technology is that the molecule binds to the target protein which is then linked to E3 ubiquitin ligase for ubiquitination modification and eventual degradation via the proteasomal pathway, we next examined whether proteasome inhibition contributes to the observed PKD-1-induced KRAS G12C protein degradation and cell viability inhibition. PS-341, a proteosome inhibitor, was used to interrogate this mechanism.

We first tested whether PS-341 alone has any inhibitory effect on the proliferation of MIA $\mathrm{PaCa}-2$ cells to rule out of nonspecific effect. PS-341 clearly demonstrated anti-proliferation effect in a dose-dependent manner (Figure 6A). In the following experiment, we chose two concentrations of PS-341, $10 \mathrm{nM}$ and $40 \mathrm{nM}$, which alone did not significantly affect cell proliferation. When studied as a pretreatment, PS-341 partially prevented the effect of PKD-1 in MIA PaCa- 2 cells with the $\mathrm{IC}_{50}$ value of PKD-1 increasing from $0.599 \mu \mathrm{mol} / \mathrm{L}$ (without PS-341 treatment) to $0.885 \mu \mathrm{mol} / \mathrm{L}$ (10 nM PS-341 treatment) and $0.856 \mu \mathrm{mol} / \mathrm{L}$ ( $40 \mathrm{nM} \mathrm{PS}-341$ treatment) (Figure 6B). The blockade was most apparent at lower PKD-1 concentrations.

This result suggests that the efficacy of PKD- 1 is diminished by proteasome inhibition and that the effect of PKD-1 against $\mathrm{PaCa}-2$ tumor cell proliferation is at least partially mediated by promoting proteasomal degradation of KRAS G12C protein.

A

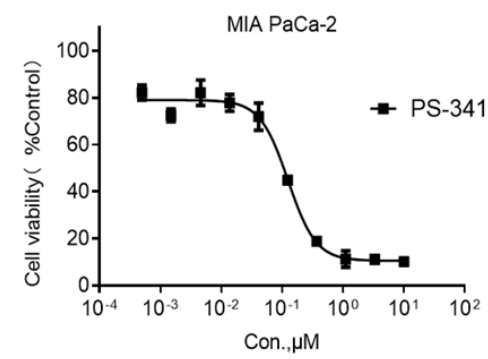

B.

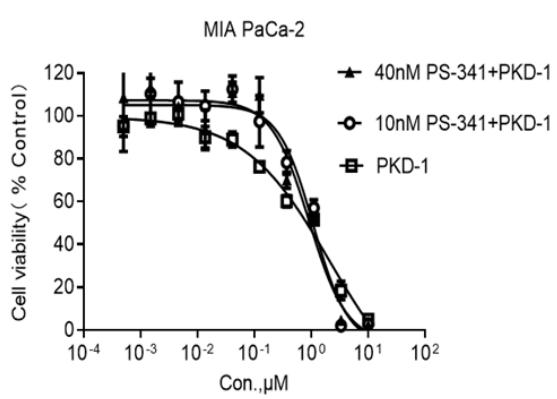

Figure 6 A. Effects of PS-341 on cell proliferation activity; B. Effect of PS-341 on the inhibitory activity of PKD-1.

\subsection{Effect of PKD-1 on the MIA PaCa-2 cell cycle}

Because PKD-1 was able to significantly inhibit cell proliferation (Figure 5), we next examined whether PKD-1 treatment could lead to cell cycle arrest, a process that is opposite to cell proliferation. Using flow cytometry, we found that the cell ratio in G0/G1 phase in MIA $\mathrm{PaCa}-2$ cells was $50.1 \%$ when there was no drug treatment (Figure 7A). In contrast, the ratio of the cells in G0/G1 phase increased dose-dependently with increasing PKD-1 concentrations, reaching $61.7 \%$ (Figure 7B) and 66.5\% (Figure 7C) at PKD-1 drug concentrations of 0.1 and 1 $\mu \mathrm{mol} / \mathrm{L}$, respectively. These results indicate that PKD-1 can lead to cell cycle arrest in MIA $\mathrm{PaCa}-2$ cells. 

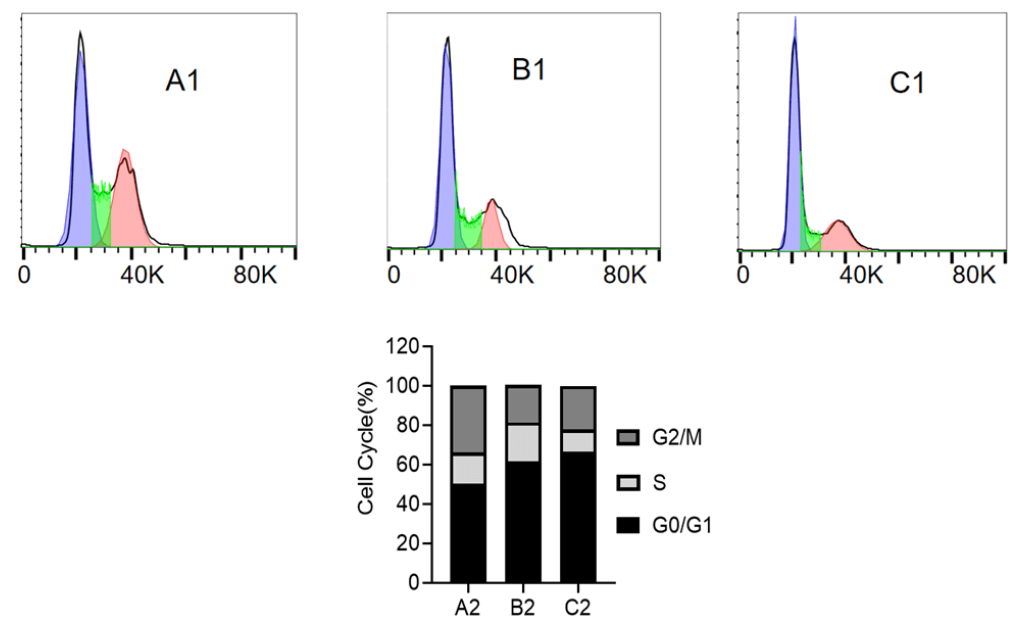

Figure 7 PKD-1 induced cell cycle arrest in G0/G1 phase. A1, A2: Control group; B1, B2: $0.1 \mu \mathrm{mol} / \mathrm{L}$ PKD-1 group; C1, C2: $1 \mu \mathrm{mol} / \mathrm{L}$ PKD-1 group.

\subsection{Effect of PKD-1 on apoptosis in MIA PaCa-2 cells}

Finally, we examined whether PKD-1 treatment could lead to cellular apoptosis. Hoechst 33258 staining was used to observe the morphological changes of cell apoptosis. As can be seen in Figure 8, increasing dose of PKD-1 dose-dependently increased the chromatin condensation as reflected by fluorescence aggregation. At the concentration of $0.1 \mu \mathrm{mol} / \mathrm{L}, \mathrm{PKD}-1$ treatment led to high percentage ( $>60 \%$ ) of apoptotic cells (Figure 8 ), indicating that PKD-1 was able to induce apoptosis in MIA PaCa-2 cells.

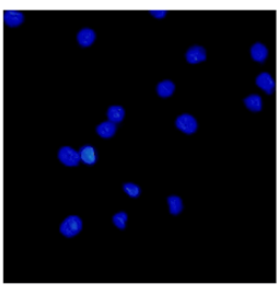

A 1

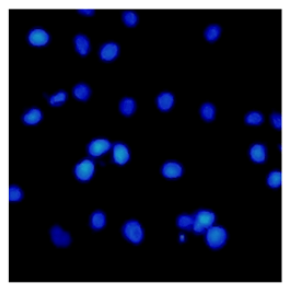

B1

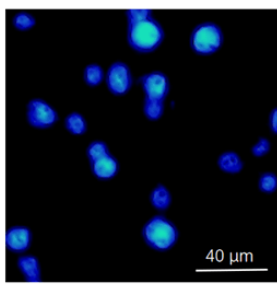

C1

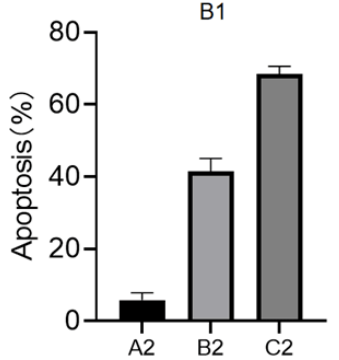

Figure 8 The cell cycle count. PKD-1, A1, A2: control group; B1, B2: $0.1 \mu \mathrm{mol} / \mathrm{L}$ group; $\mathrm{C} 1, \mathrm{C} 2: 1 \mu \mathrm{mol} / \mathrm{L}$ group; The data are expressed as the mean $\pm \mathrm{SD}(\mathrm{n}=3)$, Compared with the control group, ${ }^{*} p<0.05$.

\section{Discussion and conclusion}

The primary findings of this study were that PKD-1 was able to bind to and promote the degradation of KRAS G12C protein, inhibit cell proliferation, promote cell cycle arrest and apoptosis in the pancreatic cancer cell line $\mathrm{PaCa}-2$. In addition, the results showed that these effects of PKD-1 were at least partially mediated by promoting proteasomal degradation of KRAS G12C protein. Combined, these results suggest that PKD-1 could be a useful therapy against pancreatic cancer and that the PROTAC technology is a viable approach to develop targeted therapy for pancreatic cancer.

The RAS genes are the most frequently mutated family of oncogenes in cancer. Of these, KRAS mutations are found in the majority of pancreatic cancer cases. KRAS G12C is a specific KRAS sub-mutation, resulting from the substitution of glycine by cysteine at codon 12, and 
accounts for approximately $44 \%$ of all KRAS mutations. Developing targeted treatment for pancreatic cancer caused by KRAS G12C mutations [18] has been an emerging strategy in recent years with the PROTAC technique.

PROTAC technology consists of a POI ligand, linker, and E3 ligase ligand. The PROTAC molecule directs the 26S proteasome to target proteins for targeted degradation by ubiquitinating the target protein [19]. The first reported successful PROTAC-induced endogenous KRAS G12C degradation in several KRAS-mutant cancer cell lines developed a compound LC-2 which used MRTX-849 as the POI ligand and demonstrated high efficacy to induce sustained KRAS G12C degradation [15]. In this study, we chose PKD-1 for all cellular assays which is different from LC-2 in that the POI ligand was structurally different from MRTX-849 and which demonstrates good $(>70 \%)$ degradation efficacy in SW1573 cells [15]. We first analyzed the binding ability of PKD-1 to KRAS and its ability to degrade KRAS proteins, after which we performed assays on the biological functions of PKD-1, including its effect on the proliferative effect of pancreatic cancer cells (MIA PaCa-2), its effect on the cell cycle and its effect on apoptosis. The results of the binding assay showed that PKD-1 was able to produce binding to KRAS proteins with an $\mathrm{EC}_{50}$ of $144.7 \mathrm{nmol} / \mathrm{L}$, similar to the ability of MRTX-849. In the Western blotting assay, it showed that the molecular weight of KRAS protein increased with the increase of the PKD-1 concentration, demonstrating a clear upward shift of the KRAS G12C protein band. This result was similar to the reported effect of MRTX-849 [15] and clearly showed that PKD-1 was able to bind to KRAS G12C protein. In the time course study, PKD-1 was found significantly and time-dependently reduced the KRAS protein level, suggesting that PKD-1 led to KRAS protein degradation for up to $72 \mathrm{~h}$.

In functional assays, PKD-1 was found to significantly inhibit the proliferation of MIA PaCa2 cells with an $\mathrm{IC}_{50}$ of $0.276 \mu \mathrm{mol} / \mathrm{L}$, similar to that of the positive drug MRTX-849. PKD-1 at concentrations of 0.1 and $1 \mu \mathrm{mol} / \mathrm{L}$ significantly promoted cell cycle arrest in the G0/G1 phase and prevented cell cycle progression, which is consistent with the observed inhibition of cell proliferation. Importantly, PKD-1 was found to significantly promote cell apoptosis in MIA PaCa-2 cells, and morphological observation found that PKD-1-treated cells showed clear nuclear sequestration. These results suggest that PKD-1 can exert the anti-proliferative effect by blocking the progression of the cell cycle and promoting apoptosis. To further investigate whether this effect of PKD-1 acts by promoting KRAS degradation, we used PS-341, an inhibitor of the proteasome, to inhibit the proteasomal process, thereby blocking the degradation process of KRAS protein. After the inhibition of $26 \mathrm{~S}$ proteasome action, although PKD-1 was able to bind to KRAS protein, the degradation of KRAS protein was significantly reduced. These results further suggest that the anti-proliferative efficacy of PKD-1 was at least partially mediated via promoting proteasome-mediated degradation of protein ubiquitination.

In summary, this study extended previous findings by showing that a PROTAC molecule, PKD-1, was able to bind to and promote the degradation of KRAS G12C protein in pancreatic cancer cells, which led to cell cycle arrest and apoptosis. The demonstrated efficacy of PKD-1 along with the reported effects of LC-2 in several cancer cell lines support the notion that PROTAC engineered molecules is a viable drug development approach to design and develop effective new therapies for targeted therapy of certain cancers such as pancreatic cancer.

\section{Author contributions}

Formal analysis: Shuai Gao and Fangxia Zou;

Funding acquisition: Jingwei Tian and Liang Ye;

Investigation: Shuai Gao, Lixia Zheng, Yunjie Wang, Xinyu Feng and Deshuai Liu;

Methodology: Shuai Gao, Yutong Mao and Liang Ye;

Project administration: Jingwei Tian and Liang Ye;

Resources: Jingwei Tian and Liang Ye;

Supervision: Fangxia Zou and Lixia Zheng;

Writing - original draft: Shuai Gao and Lixia Zheng;

Writing - review \& editing: Jingwei Tian.

\section{Funding}

The author(s) disclosed receipt of the following financial support for the research, authorship, and/or publication of this article: This work was supported by the "Taishan Industry Leading Talent Laureate" and the "Major New Drugs Research \& Development" special projects of Ministry of Science and Technology of PR China (NO. 2018ZX09303015). 


\section{Conflict of interest}

None of the authors have reported a conflict of interest.

\section{References}

[1] Goral V. Pancreatic Cancer: Pathogenesis and Diagnosis. Asian Pacific Journal of Cancer Prevention, 2015, 16(14): 5619-5624 https://doi.org/10.7314/apjcp.2015.16.14.5619

[2] Jones S, Zhang X, Parsons DW, et al. Core signaling pathways in human pancreatic cancers revealed by global genomic analyses. Science, 2008, 321(5897): 1801-1806. https://doi.org/10.1126/science.1164368

[3] Lippi G and Mattiuzzi C. The global burden of pancreatic cancer. Archives of Medical Science, 2020 16(4): $820-824$ https://doi.org/10.5114/aoms.2020.94845

[4] Park W, Chawla A and O'Reilly EM. Pancreatic cancer: a review. JAMA, 2021, 326(9): 851-862. https://doi.org/10.1001/jama.2021.13027

[5] American Cancer Society. Retrieved on December 12, 2021. https://www.cancer.org/cancer/pancreatic-cancer/detection-diagnosis-staging/survival-rates.html

[6] Lanfredini S, Thapa A and O'Neill E. RAS in pancreatic cancer. Biochemical Society Transactions, 2019, 47(4): 961-972. https://doi.org/10.1042/BST20170521

[7] Simanshu DK, Nissley DV and McCormick F. RAS Proteins and Their Regulators in Human Disease. Cell, 2017, 170(1): 17-33. https://doi.org/10.1016/j.cell.2017.06.009

[8] Rossman KL, Der CJ and Sondek J. GEF means go: turning on RHO GTPases with guanine nucleotide-exchange factors. Nature Reviews Molecular Cell Biology, 2005, 6: 167-180. https://doi.org/10.1038/nrm1587

[9] Canon J, Rex K, Saiki AY, et al. The clinical KRAS (G12C) inhibitor AMG 510 drives anti-tumour immunity. Nature, 2019, 575(7781): 217-223. https://doi.org/10.1038/s41586-019-1694-1

[10] Hallin J, Engstrom LD, Hargis L, et al. The KRAS (G12C) Inhibitor MRTX849 Provides Insight toward Therapeutic Susceptibility of KRAS-Mutant Cancers in Mouse Models and Patients. Cancer Discovery, 2020, 10(1): 54-71.

https://doi.org/10.1158/2159-8290.CD-19-1167

[11] Bond MJ, Chu L, Nalawansha DA, et al. Targeted Degradation of Oncogenic KRAS (G12C) by VHL-Recruiting PROTACs. ACS Cent Sci, 2020, 6(8): 1367-1375. https://doi.org/10.1021/acscentsci.0c00411

[12] Paiva SL and Crews CM. Targeted protein degradation: elements of PROTAC design. Current Opinion in Chemical Biology, 2019, 50: 111-119. https://doi.org/10.1016/j.cbpa.2019.02.022

[13] Salami J and Crews CM. Waste disposal - An attractive strategy for cancer therapy. Science, 2017, 355(6330): 1163-1167. https://doi.org/10.1126/science.aam7340

[14] Lin X, Xiang H and Luo G. Targeting estrogen receptor $\alpha$ for degradation with PROTACs: A promising approach to overcome endocrine resistance. European Journal of Medicinal Chemistry, 2020, 206: 112689 . https://doi.org/10.1016/j.ejmech.2020.112689

[15] Crew AP, Hornberger KR, Wang J, et al. Modulators of proteolysis and associated methods of use. WO2019/195609A2, 2019.

[16] Li DJ, Tong J, Zeng FY, et al. Nicotinic Ach alpha7 inhibits PDGF-induced migration of vascular smooth muscle cells by activating mitochondrial deacetylase sirtuin 3. British Journal of Pharmacology, 2019, 176(22): 4388-4401.

https://doi.org/10.1111/bph.14506

[17] Wang HB, Ma XJ, Ren SM, et al. A small-molecule inhibitor of MDMX activates p53 and induces apoptosis. Molecular Cancer Therapeutics, 2011, 10(1): 69-79. https://doi.org/10.1158/1535-7163.MCT-10-0581

[18] Drosten M and Barbacid M. Targeting the MAPK Pathway in KRAS-Driven Tumors. Cancer Cell, 2020, 37(4): 543-550.

https://doi.org/10.1016/j.ccell.2020.03.013

[19] Burslem GM and Crews CM. Proteolysis-Targeting Chimeras as Therapeutics and Tools for Biological Discovery. Cell, 2020, 181(1): 102-114. https://doi.org/10.1016/j.cell.2019.11.031 